\title{
High-Power Versatile Picosecond Pulse Generation from Mode-Locked Quantum-Dot Laser Diodes
}

\author{
Maria Ana Cataluna, Member, IEEE, Ying Ding, Member, IEEE, \\ Daniil I. Nikitichev, Student Member, IEEE, Ksenia A. Fedorova, Student Member, IEEE, \\ and Edik U. Rafailov, Senior Member, IEEE
}

(Invited Paper)

\begin{abstract}
This paper presents the current status of our research in mode-locked quantum-dot edge-emitting laser diodes, particularly highlighting the recent progress in spectral and temporal versatility of both monolithic and external-cavity laser configurations. Spectral versatility is demonstrated through broadband tunability and novel mode-locking regimes which involve distinct spectral bands, such as dual-wavelength mode-locking and robust, high-power wavelength bistability. Broad tunability of the pulse repetition rate is also demonstrated for an external-cavity mode-locked quantum-dot laser, revealing a nearly constant pulse peak power at the different pulse repetition rates. High-energy and low-noise pulse generation are demonstrated for low pulse repetition rates. These recent advances confirm the potential of quantum-dot lasers as versatile, compact and low-cost sources of ultrashort pulses.
\end{abstract}

Index Terms - Laser mode locking, quantum dot lasers, optical pulses, laser tuning.

\section{INTRODUCTION}

$\mathrm{S}$ EMICONDUCTOR quantum dots have been recently showing great promise for the generation of ultrashort pulses, forming the basis of very compact and efficient ultrafast laser sources [1]. Quantum dots (QD) are clusters of semiconductor material with dimensions of a few nanometers, embedded in a matrix with a higher bandgap than the QD material [2]. If one takes into account that all the three dimensions of a QD cluster are comparable or smaller than the typical de Broglie wavelength for a III-V compound

Manuscript received December 8, 2010. This work was supported by the European Community's Seventh Framework Programme (FP7/2007-2013) under grant agreement $\mathrm{n}^{\circ} 224338$ (FAST-DOT project). M. A. Cataluna also acknowledges financial support through a Royal Academy of Engineering/EPSRC Research Fellowship.

M. A. Cataluna, Y. Ding, D. I. Nikitichev, K. A. Fedorova, E. U. Rafailov are with the Photonics and Nanoscience Group, School of Engineering, Physics and Mathematics, University of Dundee, Dundee DD1 4HN, U. K. (e-mail: m.a.cataluna@ dundee.ac.uk, y.ding@dundee.ac.uk,

d.nikitichev@dundee.ac.uk, k.a.fedorova@dundee.ac.uk, e.u.rafailov@dundee.ac.uk). semiconductor (of the order of tens of nanometers), then it becomes evident that a strong three-dimensional quantum confinement of the carriers is in place. Such a strong confinement leads to dramatically different energy-momentum relations, resulting in a delta-like density of states. As such, in quantum dots the charge carriers occupy only a restricted set of energy levels. It is this strong three-dimensional carrier confinement - akin to a nearly zero-dimensionality - which strongly determines the major advantages predicted and demonstrated for QD lasers, such as low threshold current and high characteristic temperature [3]. On the other hand, the self-organised growth of quantum dots - usually through the Stranski-Krastanow growth method - leads to a Gaussian distribution of dot sizes. Due to the three-dimensional quantum confinement, such a variation in size leads to a corresponding Gaussian distribution of emission frequencies. Moreover, lattice strain may vary across the wafer, thus further affecting the energy levels in the quantum dots. Such effects lead to the inhomogeneous broadening of the gain and absorption in the QD material [3]. This is a useful phenomenon in the context of ultrafast applications, because a very wide bandwidth is available for the generation, propagation and amplification of ultrashort pulses [4], while also being extremely useful for the generation of a widely tunable output $[5,6]$. These attributes, together with the ultrafast gain and absorption recovery times exhibited by QD materials [7, 8], have been affording major advantages in ultrafast science and technology since the first demonstration of a QD two-section mode-locked laser diode in 2001 [9].

In fact, monolithic passively mode-locked QD lasers can currently surpass the performance of similar quantum-well (QW) lasers in terms of pulse duration, with pulses as short as 400fs generated directly from InAs/GaAs QD laser diodes operating in the $1.26 \mu \mathrm{m}$ waveband [10], or with pulse durations down to $312 \mathrm{fs}$ in the $1.55 \mu \mathrm{m}$ spectral band, as demonstrated with InAs/InP QD lasers [11]. QD lasers have also shown a superior performance to similar QW lasers in the case of pulse timing jitter [12, 13], achieving record-low 
values too $[14,15]$.

Temperature resilience is an important feature for most applications - in this context, it was demonstrated that stable mode-locked operation can be achieved in a QD laser at elevated temperatures up to $80^{\circ} \mathrm{C}$, and at relatively high output average powers [16]. More recently, the use of p-doped QD structures in mode-locked lasers has extended the range of bias conditions where stable mode-locking is observed at elevated temperatures, while simultaneously achieving an infinite characteristic temperature [17]. Furthermore, it has been shown that the duration of the pulses generated actually decrease with temperature $[18,19]$, which can be attributed to a faster absorber recovery time with increasing temperature most notably due to a faster thermionic escape $[19,20]$.

In this paper we report on our recent results that exploit the unique properties of $\mathrm{QD}$ materials in novel and versatile mode-locking regimes in InAs/GaAs QD edge-emitting monolithic lasers, by harnessing the spectral versatility offered by the presence of the excited-state transition (Section II) and the possibilities of QD material engineering in the development of nonidentical QD layer structures (Section III). We also present our latest investigation of external-cavity QD mode-locked lasers with variable pulse repetition rate, low noise and high pulse energy (Section IV).

\section{EXPloiting the EXcited-State Transition In NOVEL MODE-LOCKING REGIMES}

The discrete energy levels displayed by QD materials can be exploited to access distinct spectral bands in the same device, adding an extra level of functionality to QD lasers. Indeed, light emission in a QD laser can occur via the lower-energy ground-state (GS) and/or the higher-energy excited-state (ES) transitions, thus engaging quite different spectral regions [21]. Subpicosecond gain recovery has been demonstrated for both GS and ES transitions in electrically-pumped QD amplifiers [22]. Two-color pump-probe investigations of reverse-biased waveguide saturable absorbers have also revealed that the absorption recovery time for the ES transition is faster than for the GS transition under the same reverse bias conditions [23]. It is therefore quite relevant to investigate the potential for ultrashort pulse generation across both GS and ES transitions. In this context, we have previously demonstrated an optically gain-switched QD laser, where pulses were generated from both GS and ES, and where the ES pulses were shorter than those generated by GS [24]. We then demonstrated, for the first time, passive mode locking via GS $(1260 \mathrm{~nm})$ or ES $(1190 \mathrm{~nm})$ in a quantum-dot laser, at repetition frequencies of $21 \mathrm{GHz}$ and $20.5 \mathrm{GHz}$, respectively [25, 26]. The switch between these two states in the mode-locking regime was easily achieved by changing the electrical biasing conditions, thus providing full control of the operating spectral band. Subsequent demonstrations of switching between GS and ES mode-locking followed, in an external cavity configuration [27], and using the self-electro-optical effect for reverse switching (first ES, then GS mode-locking) [28]. Dual-wavelength mode-locking was previously reported for a single-section InP-based QD laser diode, with pulses being generated simultaneously at 1543 and $1571 \mathrm{~nm}$ [29]. However, these spectral bands are not ascribed to the ES/GS transitions, and the hypothesis presented by the authors is that the energy-level splitting corresponds to the Rabi oscillation frequency. In this demonstration, it is also shown that the spectral separation varies with the driving conditions [29].

We have recently achieved a dual-wavelength passive mode-locking regime where pulses are generated simultaneously from both ES $(\lambda=1180 \mathrm{~nm})$ and GS $(\lambda=1263 \mathrm{~nm})$, in a two-section GaAs-based QD laser [30, 31]. This is the widest spectral separation $(83 \mathrm{~nm})$ ever observed in a dual-wavelength mode-locked non-vibronic laser [32].

The dual-wavelength mode locking regime was achieved in a two-section, 2mm-long laser diode, with a saturable absorber length of $300 \mu \mathrm{m}$. The front and back facets were anti-reflection ( 3\%) and high-reflection ( $95 \%)$ coated, respectively. Its active region incorporated 5 layers of InAs QDs. The pulse durations in both GS and ES spectral bands (and all those referred to in this paper) were measured by a non-collinear autocorrelator based on second-harmonic generation (Femtochrome FR 103-XL).

A stable dual-wavelength mode-locking regime was found
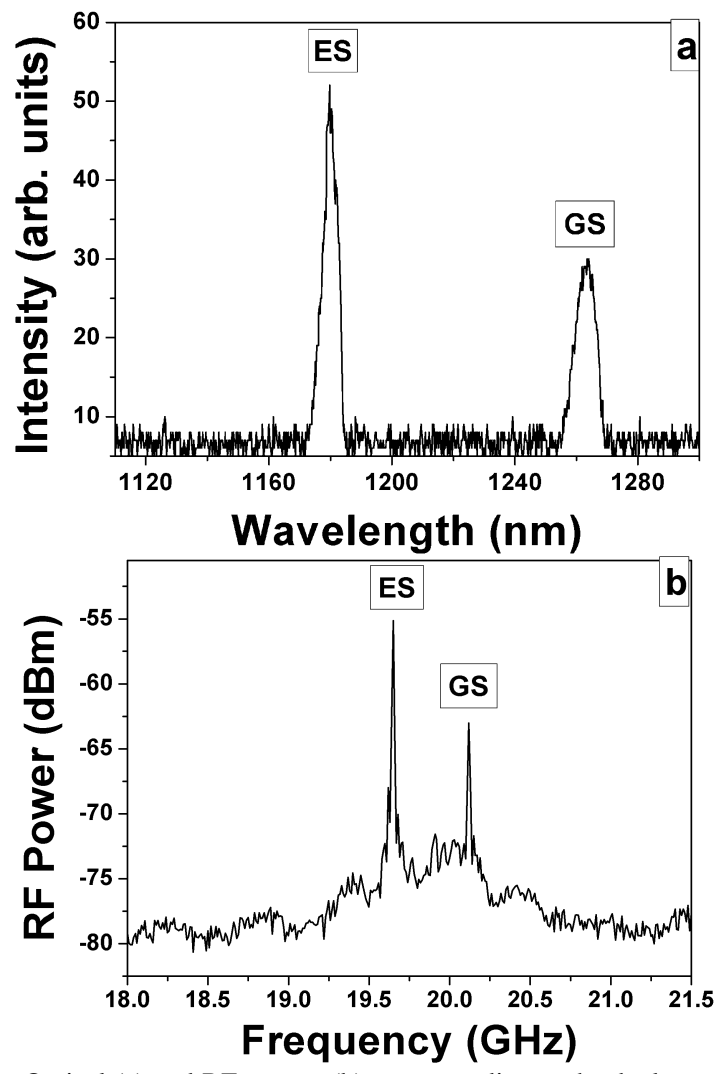

Fig. 1. Optical (a) and RF spectra (b) corresponding to the dual-wavelength mode-locking regime, for a reverse bias of $6.7 \mathrm{~V}$ and gain current injection of $400 \mathrm{~mA}$. 
in a range of bias conditions, which simultaneously satisfied the conditions for achieving mode-locking via GS and ES: higher current levels applied to the gain section (between 330 and $430 \mathrm{~mA}$ ) and values of reverse bias between $6 \mathrm{~V}$ and $10 \mathrm{~V}$ in the saturable absorber section. The excited state levels have higher degeneracy, and consequently higher saturated gain than the GS. This means that a transition from the GS to the ES can be achieved by increasing loss, which in this case can be manipulated through the increase in reverse bias applied to the saturable absorber.

The optical and RF spectra are represented in Fig. 1, for an applied current of $400 \mathrm{~mA}$ and a reverse bias of $6.7 \mathrm{~V}$. The spectral separation between the two modes results in different repetition rates, due to the dispersive nature of the laser semiconductor material, which induces different cavity roundtrip times for the propagation of the two modes. The repetition rates of the generated pulses were $19.7 \mathrm{GHz}$ and $20.1 \mathrm{GHz}$ for the ES $(\lambda=1180 \mathrm{~nm}$ with a full-width at half-maximum of $4.8 \mathrm{~nm}$, resulting in a time-bandwidth product of 8.2$)$ and GS $(\lambda=1263 \mathrm{~nm}$ with a full-width at half-maximum of $6.2 \mathrm{~nm}$, resulting in a time-bandwidth product of 7), respectively. For these bias conditions, the ES pulse duration was $7.9 \mathrm{ps}$, while the GS pulse duration was $5.9 \mathrm{ps}$. The average power was of the same order for GS and ES pulsed outputs, ranging between 20 and $30 \mathrm{~mW}$. Furthermore, the same laser also displayed, for different bias conditions, several other regimes of operation, such as modelocking via the GS or via the ES, and continuous-wave emission via the GS [31], which clearly highlights the spectral versatility that QD structures can offer.

An alternative method for achieving dual-wavelength modelocking involving the GS $(\lambda=1270 \mathrm{~nm})$ and $\mathrm{ES}(\lambda=1207 \mathrm{~nm})$ has also been subsequently demonstrated by Breuer et al. [33], by using a 3-mm multi-section laser where the active region incorporates six chirped QD layers. This mode-locking regime is achieved under low current values applied to the gain section, as well lower reverse bias - leading, however, to lower output power levels - below $4 \mathrm{~mW}$ for the GS, and less than $2 \mathrm{~mW}$ for the ES [33].

It is important to add that the development of dual- and multiple-wavelength ultrafast lasers is a research area that aims to address a number of important applications such as time-domain spectroscopy, wavelength division multiplexing and nonlinear optical frequency conversion. The spectral versatility of a QD mode-locked diode laser which is switchable between GS and ES has already demonstrated its potential as a two-band clock recovery source [34]. In this perspective, the compactness, lower cost and direct electrical pumping associated with semiconductor lasers form a set of attractive features for reducing the footprint and complexity of the aforementioned applications, with the potential to also open up new avenues in ultrafast optical processing and optical interconnects.

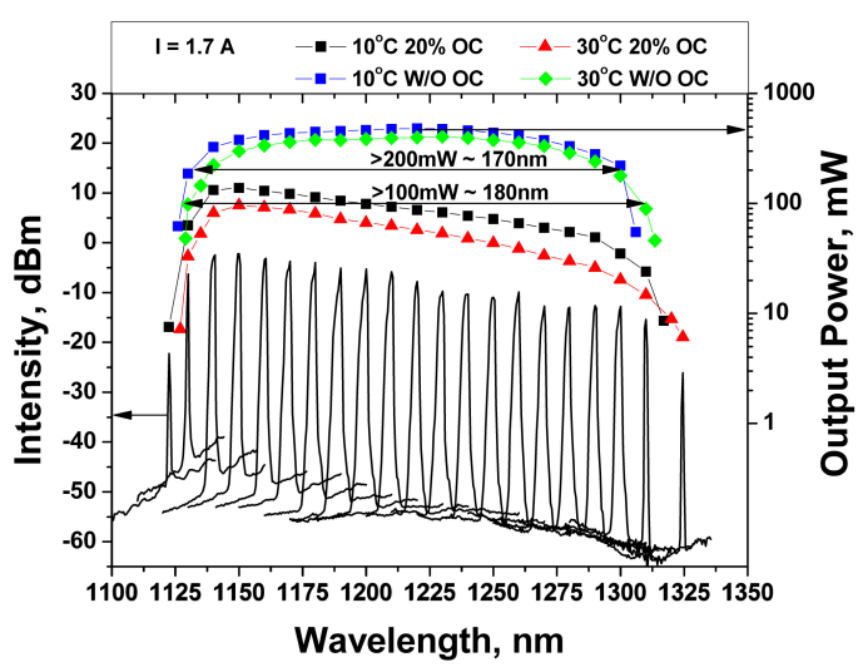

Fig. 2. Tunability range and output power achieved in a tunable quantum-dot external-cavity laser, with a $20 \%$ output coupler (OC) or without (W/O) output coupler, at different operating temperatures.

\section{WAVELENGTH-BISTABLE MODE-LOCKING REGIME}

Quantum-dot (QD) materials have shown great promise as a gain element of broadly tunable laser sources, due to the inhomogeneous broadening associated with the size dispersion of the QDs. Recently developed growth techniques can control the fabrication of InAs/GaAs QDs with different sizes, giving access to an unprecedented broad spectral range in the spectral region between $1.0 \mu \mathrm{m}$ and $1.3 \mu \mathrm{m}$. By exploiting such broad gain bandwidth, QD external-cavity diode lasers (QD-ECDL) have demonstrated impressive tuning ranges up to 202nm [6], with nearly $0.5 \mathrm{~W}$ output power in the central part of the tuning range, as illustrated in Fig. 2. Broadband tunability of up to $166 \mathrm{~nm}$ has also been demonstrated in a QD-ECDL based on InAs/InP quantum-dot materials, covering the $1.55 \mu \mathrm{m}$ spectral region [35].

We have very recently demonstrated a new approach to achieve wavelength bistability in a multi-section QD laser, by using an active region which incorporates non-identical InAs QD layers which emit at distinct wavelengths - a similar structure to that used for the broadly tunable QD laser [6]. Wavelength bistability in semiconductors lasers is an exciting area for the development of the next generation of optical communication systems and all-optical processing components such as flip-flop memory switches [36]. In the context of high-speed applications, QD mode-locked lasers have shown great potential as efficient and compact sources of low-noise and high-repetition-rate ultrashort pulses [1]. Wavelength bistability has been recently shown in passively mode-locked QD lasers with a $7.6 \mathrm{GHz}$ pulse repetition rate, using a two-section device with an active region which comprised 10 InGaAs QD layers [37, 38]. Bistability between $1166 \mathrm{~nm}$ and $1174 \mathrm{~nm}$ was achieved with power suppression ratios of $30 \mathrm{~dB}$. However, the level of output power achieved in the latter work was rather low, ranging between $0.2-0.8 \mathrm{~mW}$ for an operating 


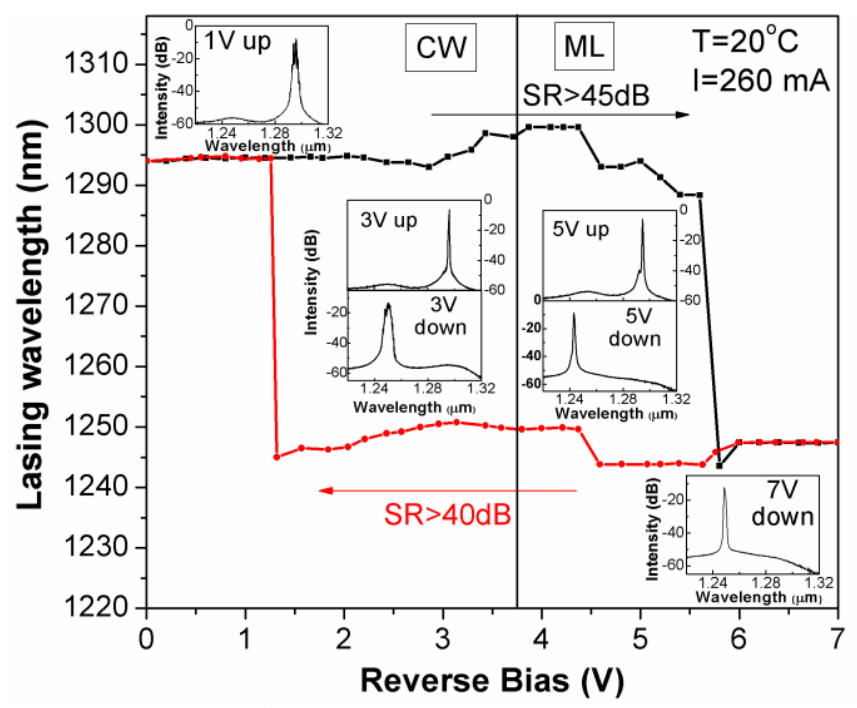

Fig. 3. Dynamics of the emission wavelength with various values of ascending and descending reverse bias. A transition between continuous wave $(\mathrm{CW})$ and mode-locked $(\mathrm{ML})$ regimes occurs at $3.75 \mathrm{~V}$. Insets: optical spectra at several operating points, depicting the suppression ratio (SR) between the modes.

temperature of $12^{\circ} \mathrm{C}$.

We have achieved robust and high-power wavelength bistability between $1245 \mathrm{~nm}$ and $1295 \mathrm{~nm}$ (the largest spectral range to our knowledge), controllable via the reverse bias applied to the saturable absorber. Depending on the range of reverse bias applied, continuous wave $(\mathrm{CW})$ or mode-locked regimes are obtained, with average powers up to $25 \mathrm{~mW}$ and $17 \mathrm{~mW}$, respectively. This output power performance represents an improvement of up to two orders of magnitude when compared with previous results $[37,38]$. A suppression ratio higher than $40 \mathrm{~dB}$ is demonstrated.

The investigated multi-section laser has a ridge waveguide width of $6 \mu \mathrm{m}$ and a total length of $4 \mathrm{~mm}$, resulting in a pulse repetition rate of $10 \mathrm{GHz}$ when mode-locked, as defined by the cavity round trip time. The device consists of multiple 1-mm-long electrically-insulated sections, each of these further divided into $300 \mu \mathrm{m}$ and $700 \mu \mathrm{m}$ sub-sections. A reverse bias is applied to the two $300 \mu \mathrm{m}$ sections placed nearer the back facet, thus forming a distributed saturable absorber with a total length of $600 \mu \mathrm{m}$. All the remaining sections are forward biased and thus form the gain section. The output facet was deep-anti-reflection coated (on the gain section side), while the back facet was high-reflection coated (on the absorber side), with reflectivities of approximately $0.1 \%$ and 95\%, respectively. The QD structure was grown on a GaAs substrate by molecular beam epitaxy. Its active region contains $10 \mathrm{InAs}$ QD layers covered by non-identical InGaAs capping layers, incorporated into $\mathrm{Al}_{0.35} \mathrm{Ga}_{0.65} \mathrm{As}$ cladding layers. This growth technique results in increased indium segregation into the quantum dots that have thicker capping layers, and as such, the average size of the quantum dots capped with thicker layers becomes larger, with an associated shift in spectral emission. As a result, this QD structure comprised 3 QD layers with an emission spectrum approximately centered at $1211 \mathrm{~nm}, 3 \mathrm{QD}$ layers at $1243 \mathrm{~nm}$ and 4 QD layers at $1285 \mathrm{~nm}$ (the concept of
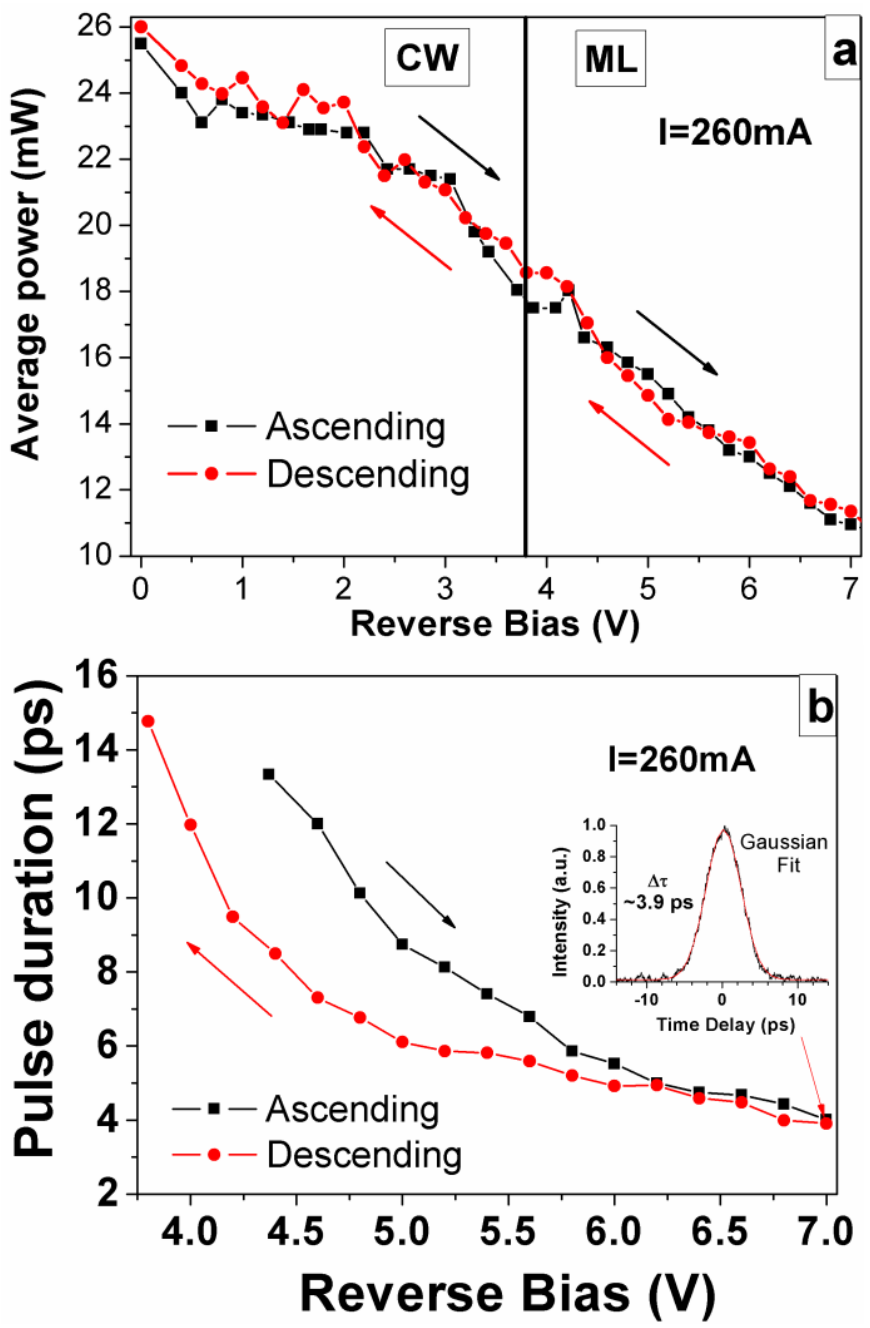

Fig. 4. (a) Output power and (b) pulse duration in the ascending and descending directions, for a fixed applied current of $260 \mathrm{~mA}$ in the gain section. Inset: autocorrelation trace corresponding to the shortest pulse measured in the bistability regime.

this structure epitaxy being similar to the one described in Ref. [6], albeit with a slight modification in one group of QD layers). The laser's operating temperature was maintained at $20^{\circ} \mathrm{C}$.

For a fixed current of $260 \mathrm{~mA}$ applied to the gain section, the reverse bias was varied between $0 \mathrm{~V}$ and $7 \mathrm{~V}$, in both ascending and descending directions. As depicted in Fig. 3, a wide wavelength bistability region was obtained, between $1 \mathrm{~V}$ and $6 \mathrm{~V}$, with the widest spectral separation of $50 \mathrm{~nm}$ being achieved between the two bands. Remarkably, the power suppression ratio was higher than $45 \mathrm{~dB}$ and $40 \mathrm{~dB}$ in the ascending and descending direction, respectively. This is demonstrated also in the insets of Fig. 3, where representative optical spectra are depicted at several points of the operation curve. A transition in the laser operation mode - between CW and mode-locking - was found for a reverse bias of $3.75 \mathrm{~V}$, for both ascending and descending directions. As such, both CW and mode-locked regimes can be accessed for both wavelengths. The output power for both directions is shown in Fig. 4 a), where there is not a very noticeable difference between the ascending and descending directions. Moreover, a significantly higher power between $10 \mathrm{~mW}$ and $17 \mathrm{~mW}$ was 
achieved under mode-locked operation, in contrast with 0.2$0.8 \mathrm{~mW}$ previously reported. The variation of the pulse duration for both increasing and decreasing reverse bias is represented in Fig 4 b). As it can be observed, the pulse duration trends reveal also a hysteresis behavior, as the pulse duration for the $1295 \mathrm{~nm}$ spectral band is longer than for the $1245 \mathrm{~nm}$ spectral band. The time-bandwidth product varied between from 6 and 1.4 for the $1295 \mathrm{~nm}$ band, as the reverse bias increased; whereas for the $1245 \mathrm{~nm}$ band, the timebandwidth product varied between 1.1 and 8.4, for a decreasing reverse bias. Under the same bias conditions, shorter pulse durations were measured for the $1245 \mathrm{~nm}$ spectral band in practically all points. The mechanism underlying the wavelength bistability results mainly from the interplay between gain and absorption for the different spectral bands. This topic will be subject to further investigation.

This new approach could potentially have a high impact in all-optical processing and optical communications - it is important to stress that the spectral range here reported is important for these applications, due to the minimum dispersion that optical fibers exhibit in that range.

\section{EXTERNAL-CAVITY MODE-LOCKING}

The typically low threshold current density and low optical losses in QD lasers have enabled the fabrication of long-cavity and extremely high wall-plug efficiency lasers with pulse repetition rates as low as $5 \mathrm{GHz}$, such as reported in [18]. There are, however, a number of applications where moving towards even lower repetition rates is highly desirable, as for example in biomedical imaging modalities which require the use of fluorescent markers - with typical relaxation times between hundreds of picoseconds and a few nanoseconds [39]. In order to decrease the repetition rate beyond the limits of what is achievable with monolithic QD mode-locked lasers, an external-cavity configuration is necessary [40, 41, 42, 13]. Although the footprint of the laser system will naturally increase, some flexibility is afforded through the possibility of tunable operation [27] or appropriate dispersion compensation which can be incorporated in the external-cavity configuration by the introduction of appropriate optical elements $[42,13]$. In our previous work, we demonstrated a QD external-cavity mode-locked laser (QD-ECMLL) incorporating a QD semiconductor saturable absorber mirror (SESAM) [41], generating pulse repetition rates ranging from $350 \mathrm{MHz}$ to 1.5 $\mathrm{GHz}$, with average output power up to $27 \mathrm{~mW}(860 \mathrm{MHz})$. In a more recent investigation [42], a QD-ECMLL is demonstrated with pulse repetition rate as low as $310 \mathrm{MHz}$, albeit with a modest pulse-energy of $\sim 0.45 \mathrm{pJ}$ (which was shown to be independent of the repetition rate). The highest peak power achieved after intra-cavity pulse compression was $0.41 \mathrm{~W}$ [42]. In this section we present our latest results achieved with a QD-ECMLL based on a two-section superluminescent diode chip. The QD chip was grown by molecular beam expitaxy (MBE) on an $\mathrm{n}^{+}$-GaAs (100) substrate which contained 10

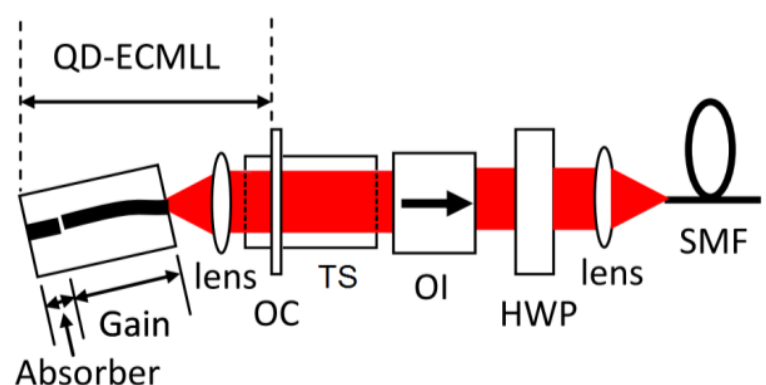

Fig. 5. Configuration of the quantum-dot external-cavity mode-locked laser. OC: output coupler; TS: translation stage; OI: optical isolator; HWP: half wave plate; SMF: single-mode fiber.

layers of self-assembled InAs/GaAs quantum dots. The waveguide in gain section is bent and terminated at an angle of $7^{\circ}$ relative to the cleaved facet, in combination with antireflection (AR) coating $\left(\mathrm{R} \sim 10^{-5}\right)$, while the back facet was high-reflection (HR) coated (R 95\%). The total chip length is $4 \mathrm{~mm}$, with a $600-\mu \mathrm{m}$-long saturable absorber section placed near the back facet. An output coupler of variable transmissivity was used for the external cavity facet. A simplified schematic of the QD-ECMLL is depicted in Fig.5.

\section{A. High peak power and pulse energy}

Using an output coupler of $96 \%$ transmissivity as the external front facet, we have demonstrated very recently the generation of high-peak-power picosecond pulses without the use of any pulse compression and optical amplification [43]. For a pulse repetition rate of $1.14 \mathrm{GHz}$, an average power of $23.2 \mathrm{~mW}$ and a peak power of $1.5 \mathrm{~W}$ with pulse duration of 13.6ps were achieved (corresponding to a 20.4-pJ pulse energy). This represents the highest peak power achieved directly from a $1.3-\mu \mathrm{m}$ waveband ECMLL. In this case, a time-bandwidth product of 3.02 can be calculated taking into account the mode-locking lasing wavelength of $1274 \mathrm{~nm}$ with $1.2-\mathrm{nm}$ full-width at half-maximum $(-7.2 \mathrm{~V}, 457 \mathrm{~mA})$. Remarkably, a minimum time-bandwidth product of 1.01 was obtained under $7.2-\mathrm{V}$ reverse bias and $330-\mathrm{mA}$ forward current with a pulse duration of $8.4 \mathrm{ps}$. Stable mode-locking with an average power up to $60 \mathrm{~mW}$ was also obtained at a repetition frequency of $2.4 \mathrm{GHz}$, which corresponds to a $25-\mathrm{pJ}$ pulse energy - a 55-fold increase in the pulse energy when compared to the demonstration in [42].

\section{B. Mode-locking trends with operation conditions}

Further investigations were carried out with this QD-ECMLL configuration at lower pulse repetition rates, using for this purpose a 53\% output coupler, which generally lead to lower output power, but also lower threshold current 

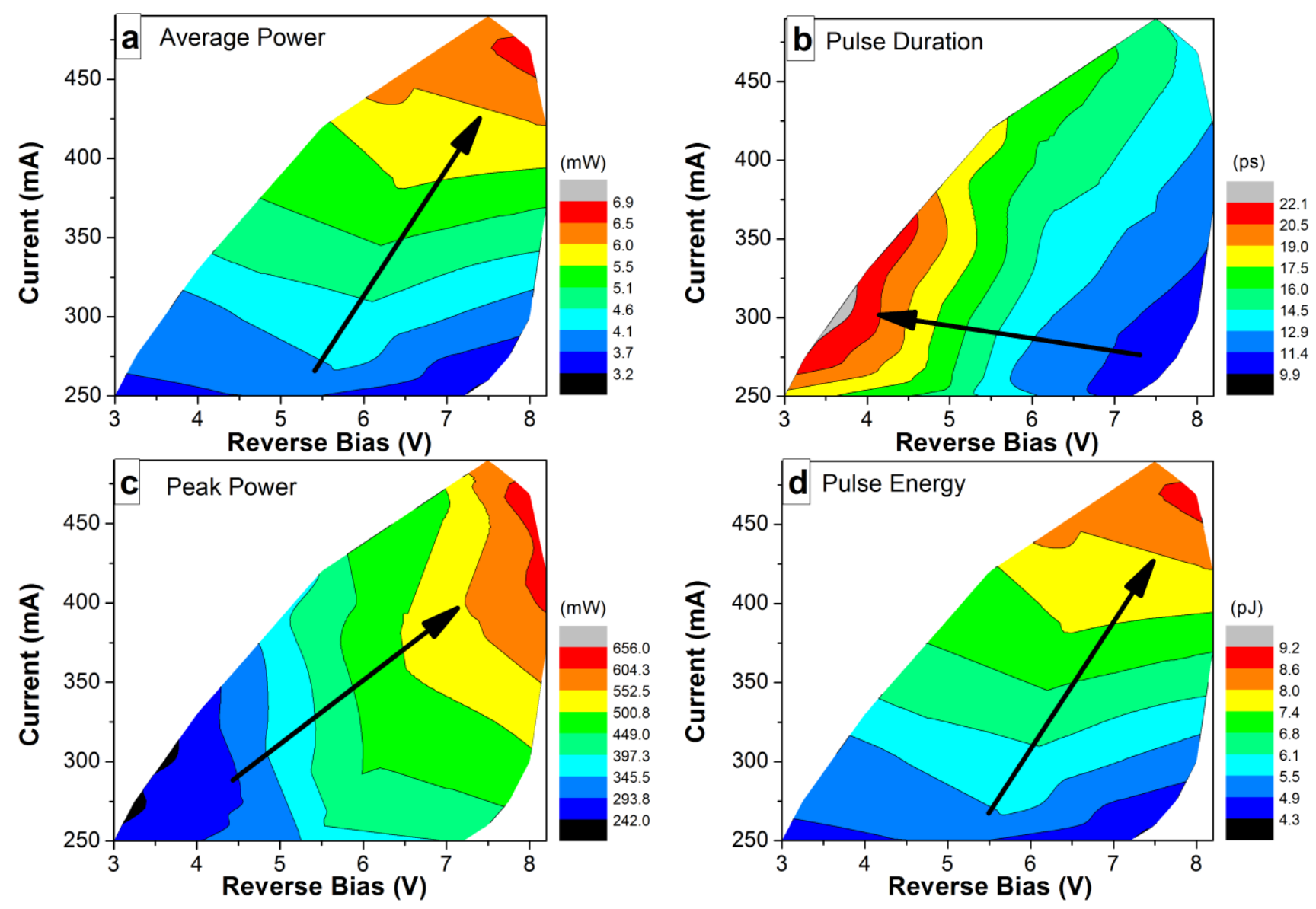

Fig. 6. (a) Average power, (b) pulse duration, (c) peak power and (d) pulse energy mapping of stable fundamental mode-locking regimes with a pulse repetition rate of $750 \mathrm{MHz}$. The arrow on the map indicates the increasing direction.

than with the $96 \%$ output coupler. It was found that such higher feedback strength was instrumental for achieving stable fundamental mode-locked operation with pulse repetition rates lower than $1 \mathrm{GHz}$. The length of the laser cavity was then adjusted such that the resulting pulse repetition rate was $750 \mathrm{MHz}$. The trends of average power, pulse energy, peak power and pulse duration with variable bias conditions were assessed within the full range where stable mode-locking was observed, as depicted in Fig. 6.

It was found that high average power (and pulse energy) can be achieved for high reverse bias and high gain current values. On the other hand, shorter pulse durations can be obtained for higher reverse bias with a low gain current - however, this bias combination leads to quite a low average power. These trends are similar to those observed in monolithic QD mode-locked laser diodes $[44,13]$, being the result of the interplay between absorption and gain dynamics. Indeed, the pulse duration decrease with increasing reverse bias can be accounted mainly by the corresponding decrease of the absorption recovery time, as has been shown by pump-probe investigations [8]. For a given applied current, this would lead to a reduction of the net gain window, with a more significant shaping of the trailing edge and pulse duration decrease.
Overall, the trends of average power and pulse duration lead to a region of higher peak power which can be achieved under high reverse bias and high current operation, as shown in Fig. $6 \mathrm{c})$.

Regarding the limits of mode-locking stability in this QD-ECMLL, it was also observed that under low reverse bias conditions, fundamental mode-locking can only be attained under low current values applied to the gain section. Moreover, for increasing values of current applied to the gain section, the region of robust fundamental mode-locking became narrower, as the laser displayed an increasing tendency for operating in a harmonic mode-locking regime, rather than in stable fundamental mode-locking. Such behaviour is in agreement with previous results reported by Viktorov et al. [45]. In this reference, the transition from fundamental mode-locking to double and triple pulsing was investigated experimentally and theoretically in monolithic QD passively mode-locked lasers, and it was shown that this transition is facilitated for higher injection current and longer cavity lengths.

\section{Trends with the pulse repetition rate}




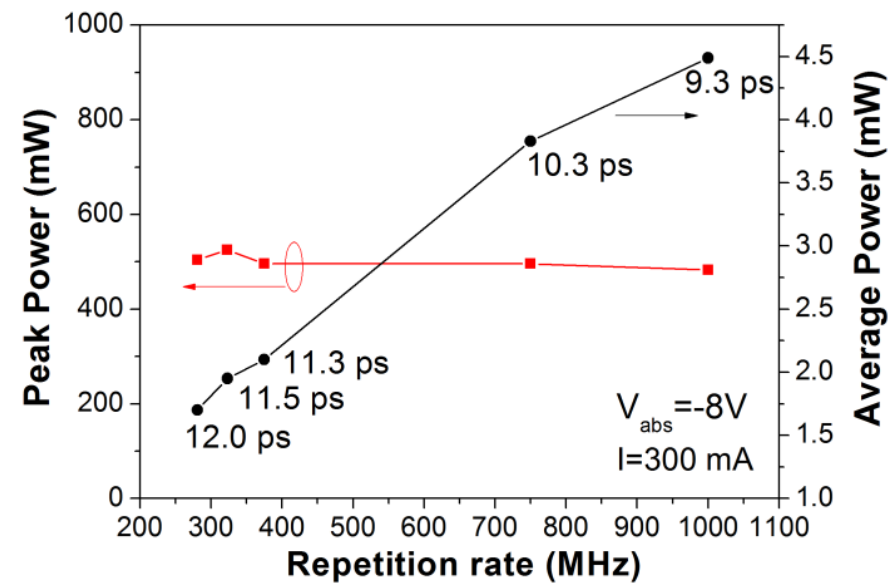

Fig. 7. Variation of the peak and average power for different pulse repetition rates, under the same bias conditions. The pulse duration achieved is indicated for each point.

By setting up the $53 \%$ output coupler on a long-travel translation stage, we were able to vary the length of the optical cavity and investigate the performance of the QD-ECMLL for a pulse repetition rate ranging between $1 \mathrm{GHz}$ and $281 \mathrm{MHz}$. For different external cavity lengths, the average power was found to be approximately proportional to the repetition frequency, as represented in Fig. 7. This seems to imply that the constant pulse energy required to saturate the absorber is independent of repetition rate under certain operation conditions, which is consistent the conclusions reported in [13], where there is mention of a constant pulse energy for a harmonic mode-locked monolithic laser with pulse repetition rates ranging between $39 \mathrm{GHz}$ and $237 \mathrm{GHz}$. Furthermore, the peak power $(\sim 0.5 \mathrm{~W})$ was also relatively constant for the different pulse repetition rates, as also evidenced in Fig. 7. The pulse duration was seen to slightly increase from $9.3 \mathrm{ps}$ to $12 \mathrm{ps}$, as the repetition rate decreased from $1 \mathrm{GHz}$ to $281 \mathrm{MHz}$. For $281 \mathrm{MHz}$ repetition rate, the optical spectrum was centred at $1268 \mathrm{~nm}(-8 \mathrm{~V}, 300 \mathrm{~mA})$, with a full-width at half-maximum of $4 \mathrm{~nm}$, resulting in a time-bandwidth product of 8.95 .

The limit to the lowest possible repetition rate achievable with a semiconductor gain material is strongly linked with its spontaneous recombination time - which for QD materials is of the order of magnitude of $\sim 1 \mathrm{~ns}$. As such, if the cavity length is extended to the point where the inter-pulse period is much longer than this recombination time, the mode-locking regime may cease to be stable as the spontaneous emission becomes a more predominant effect, as observed by a number of research groups [41, 13].

It is therefore noteworthy to stress that in the QD-ECMLL here investigated, the achieved repetition rate of $281 \mathrm{MHz}$ is in fact the lowest reported up until now for an external-cavity QD mode-locked laser diode.

\section{Low noise operation}

QD mode-locked lasers have been gaining status as

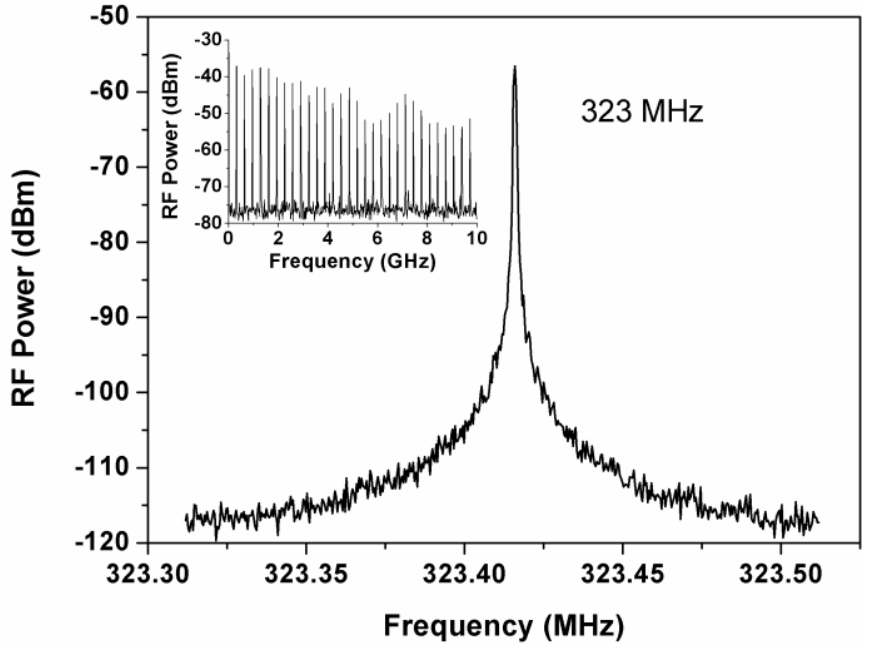

Fig. 8. RF spectrum corresponding to stable mode-locked operation with a $323 \mathrm{MHz}$ pulse repetition rate, with a $-3 \mathrm{~dB}$ linewidth of $\sim 300 \mathrm{~Hz}$ (obtained after Lorentzian fit). The resolution and video bandwidth for this acquisition were $30 \mathrm{~Hz}$ and $3 \mathrm{~Hz}$, respectively. Inset: RF spectrum with a $10 \mathrm{GHz}$ span.

low-noise sources because of the presence of discrete levels and low internal losses, enabling low threshold current densities and reduced values of amplified spontaneous emission coupled to the wavelength of operation, when compared with other higher-dimensional materials [46]. Indeed, spontaneous fluctuations in the number of photons represent one of the major sources of timing jitter, as it imparts random fluctuations in the index of refraction, thereby affecting the round-trip time and thus the timing of the pulses. In the case of external-cavity mode-locked lasers, thermal and mechanical instabilities can also contribute to timing jitter. But if these instabilities are overcome, an improvement in the phase noise and timing jitter is expected in a stable external-cavity laser when comparing to its monolithic counterpart, because the active waveguide occupies only a fraction of the optical cavity [47].

Record-low noise performance has been achieved by Delfyett's group, using a QD laser in an external ring-cavity configuration with active harmonic mode locking to generate ultrashort pulses at a repetition rate of $12.8 \mathrm{GHz}$ (via a Mach-Zender intensity modulator) [14]. The external-cavity configuration, together with the electrical synchronization allowed by active mode locking, resulted in very low residual integrated timing jitter values of $7.5 \mathrm{fs}$ (in the range between $1 \mathrm{~Hz}$ and $10 \mathrm{MHz}$ ), limited only by the driving synthesizer noise. This outstanding result represents the lowest timing jitter reported so far for mode-locked semiconductor lasers, thereby confirming the importance of QD materials in the pursuit of low-noise pulsed laser sources.

On the extreme of lower repetition rates, the expectation is that the increasingly dominant spontaneous emission contribution could start to play a more significant role and increase the noise content of the pulsed output. Our recent findings show that, even for low repetition rates, the RF spectrum can still exhibit a very narrow linewidth, as depicted in Fig. 8. Operating under a pulse repetition rate of $323 \mathrm{MHz}$ 
(corresponding to an inter-pulse period of $3.1 \mathrm{~ns}$ ), the laser exhibited an $-3 \mathrm{~dB}$ RF linewidth of $300 \mathrm{~Hz}$, measured after fitting a Lorentzian function to the RF spectrum. This is a remarkable result, when compared to the lowest RF linewidth to be reported for a passively mode-locked monolithic laser $500 \mathrm{~Hz}$, for a pulse repetition rate of $10 \mathrm{GHz}$ [48], which in fact was also based in a QD structure. Our results therefore reinforce the current view that QD lasers offer major advantages for low-noise operation, for both high and low pulse repetition rates.

\section{OUTLOOK: TOWARDS HIGHER POWER LEVELS}

In order to boost the average and peak power achievable directly from the mode-locked laser diodes, post-amplification can be implemented using compact semiconductor optical amplifiers [40, 49]. Furthermore, the use of post-amplification allows for the independent control of the pulse and power characteristics.

An alternative approach to achieve pulse generation at higher output powers is by designing edge-emitting lasers with a flared or tapered geometry [50]. Tapered lasers include a straight section - which performs the function of spatial filter in the cavity - and a tapered section with increasing width, which provides high power. Therefore, tapered lasers are a good solution to provide single spatial mode beams with high power and good beam quality. In addition to being a spatial filter, all or part of the straight section can also be used as a saturable absorber, if a reverse bias voltage is applied to it. Due to the fact that the beam mode size in the saturable absorber section is much smaller than in the gain section, the ratio between the gain and absorption saturation energies is greatly enhanced, as the saturation energy scales with the area of the mode. This enhances the pulse formation mechanisms and allows for better pulse shaping and shortening, while the higher gain saturation level further relieves the effect of pulse broadening in the gain section while also assisting in the generation of higher power pulses.

In 2006, Thompson et al. demonstrated the generation of pulses as short as 780fs, by using of a tapered waveguide configuration in a two-section InGaAs QD laser [44]. Further optimization of the saturable absorber length later resulted in the demonstration of sub-picosecond pulses with average power of $15.6 \mathrm{~mW}$ and peak power of $2.25 \mathrm{~W}$ (at $12^{\circ} \mathrm{C}$ ) [13]. Very recently, we have demonstrated the highest peak power (3.6W) ever achieved from a monolithic passively mode-locked quantum-dot laser based on a gain guided tapered configuration, with a corresponding pulse duration of $3.2 \mathrm{ps}$ [51]. Picosecond pulse generation with high average power of $209 \mathrm{~mW}$ was also demonstrated from the same laser, which is more than one order of magnitude higher than previous results. It is anticipated that post-compression of these pulses would result in relatively high values of peak power $(>20 \mathrm{~W})$. We believe that the tapered laser approach is a very promising route to achieve high-peak power pulses, and we genuinely believe that many related developments will take place in the near future.

\section{CONCLUSION}

This paper outlined our recent progress in the development of compact and efficient mode-locked laser diodes based on quantum-dot materials. We have shown how the use of the excited-state transition as an additional degree of freedom in QD lasers can be harnessed for a dual-wavelength mode-locking regime that engages both ground and excited-state spectral bands. The latest developments in QD material engineering have also enabled the growth of laser active regions incorporating non-identical QD layers with controllable central wavelength emission and spectral width. Such endeavours have made possible the demonstration of widely tuneable external cavity QD laser with high output power, as well as novel mode-locking regimes with robust and electronically controllable high-power wavelength bistability.

Finally, our latest results achieved with a QD-ECMLL have demonstrated the potential for high-energy, low-noise pulse generation and the lowest pulse repetition rate to date.

The exploitation of these results opens up many possibilities for the development of simultaneously ultrafast and widely tunable compact and efficient laser sources, thus making full use of the potential for temporal and spectral versatility that QD lasers can offer.

\section{ACKNOWLEDGMENT}

The authors would like to acknowledge Dr. Igor Krestnikov and Dr. Daniil Livshits for the growth and fabrication of the laser chips (Innolume $\mathrm{GmbH}$ ).

\section{REFERENCES}

[1]
E. U. Rafailov, M. A. Cataluna, and W. Sibbett, "Mode-locked quantum-dot lasers," Nat. Photon., vol. 1, pp. 395-401, 2007. D. Bimberg, M. Grundmann, and N. N. Ledentsov, Quantum Dot Heterostructures. Chichester: John Wiley \& Sons Ltd., 1999. V. M. Ustinov, A. E. Zhukov, A. Y. Egorov, and N. A. Maleev, Quantum Dot Lasers. New York: Oxford University Press, 2003. E. U. Rafailov, P. Loza-Alvarez, W. Sibbett, G. S. Sokolovskii, D. A. Livshits, A. E. Zhukov, and V. M. Ustinov, "Amplification of femtosecond pulses over by $18 \mathrm{~dB}$ in a quantum-dot semiconductor optical amplifier," IEEE Photon. Technol. Lett., vol. 15, pp. 1023-1025, 2003.

P. M. Varangis, H. Li, G. T. Liu, T. C. Newell, A. Stintz, B. Fuchs, K. J. Malloy, and L. F. Lester, "Low-threshold quantum dot lasers with $201 \mathrm{~nm}$ tuning range," Electron. Lett., vol. 36, pp. 15441545, Aug 2000.

K. A. Fedorova, M. A. Cataluna, I. Krestnikov, D. Livshits, and E. U. Rafailov, "Broadly-Tunable High-Power InAs/GaAs QuantumDot External-Cavity Diode Lasers," Opt. Express, vol. 18, pp. 19438-19443, 2010.

P. Borri, S. Schneider, W. Langbein, and D. Bimberg, "Ultrafast carrier dynamics in InGaAs quantum dot materials and devices," $J$. Opt. A, vol. 8, pp. S33-S46, Apr 2006. 
D. B. Malins, A. Gomez-Iglesias, S. J. White, W. Sibbett, A. Miller, and E. U. Rafailov, "Ultrafast electroabsorption dynamics in an InAs quantum dot saturable absorber at $1.3 \mu \mathrm{m}, "$ Appl. Phys. Lett., vol. 89, pp. 171111-3, 2006.

[9] X. D. Huang, A. Stintz, H. Li, L. F. Lester, J. Cheng, and K. J. Malloy, "Passive mode-locking in $1.3 \mu \mathrm{m}$ two-section InAs quantum dot lasers," Appl. Phys. Lett., vol. 78, pp. 2825-2827, May 2001.

[10] E. U. Rafailov, M. A. Cataluna, W. Sibbett, N. D. Il'inskaya, Y. M. Zadiranov, A. E. Zhukov, V. M. Ustinov, D. A. Livshits, A. R. Kovsh, and N. N. Ledentsov, "High-power picosecond and femtosecond pulse generation from a two-section mode-locked quantum-dot laser," Appl. Phys. Lett., vol. 87, pp. 81107-3, 2005.

[11] Z. G. Lu, J. R. Liu, S. Raymond, P. J. Poole, P. J. Barrios, and D. Poitras, "312-fs pulse generation from a passive C-band InAs/InP quantum dot mode-locked laser," Opt. Express, vol. 16, pp. 10835-10840, Jul 72008.

[12] L. Zhang, L. Cheng, A. L. Gray, S. Luong, J. Nagyvary, F. Nabulsi, L. Olona, K. Sun, T. Tumolillo, R. Wang, C. Wiggins, J. Zilko, Z. Zou, P. M. Varangis, H. Su, and L. F. Lester, "Low timing jitter, $5 \mathrm{GHz}$ optical pulses from monolithic two-section passively mode-locked 1250/1310 nm quantum dot lasers for high-speed optical interconnects," in Optical Fiber Communication Conference, Anaheim, USA, 2005.

[13] M. G. Thompson, A. R. Rae, M. Xia, R. V. Penty, and I. H. White, "InGaAs Quantum-Dot Mode-Locked Laser Diodes," IEEE J. Sel. Topics Quantum Electron., vol. 15, pp. 661-672, May-Jun 2009.

[14] M.-T. Choi, J.-M. Kim, W. Lee, and P. J. Delfyett, "Ultralow noise optical pulse generation in an actively mode-locked quantum-dot semiconductor laser," Appl. Phys. Lett., vol. 88, pp. 131106-3, 2006.

[15] M. G. Thompson, D. Larson, A. Rae, K. Yvind, R. Penty, I. H. White, J. Hvam, A. Kovsh, S. Mikhrin, D. A. Livshits, and I. Krestnikov, "Monolithic hybrid and passive mode-locked $40 \mathrm{GHz}$ quantum dot laser diodes," in 32nd European Conference on Optical Communication, Nice, France, 2006.

[16] M. A. Cataluna, E. U. Rafailov, A. D. McRobbie, W. Sibbett, D. A. Livshits, and A. R. Kovsh, "Stable mode-locked operation up to $80{ }^{\circ} \mathrm{C}$ from an InGaAs quantum-dot laser," IEEE Photon. Technol. Lett., vol. 18, pp. 1500-1502, 2006.

[17] G. Fiol, C. Meuer, H. Schmeckebier, D. Arsenijevic, S. Liebich, M. Laemmlin, M. Kuntz, and D. Bimberg, "Quantum-Dot Semiconductor Mode-Locked Lasers and Amplifiers at $40 \mathrm{GHz}$," IEEE J. Quantum Electron., vol. 45, pp. 1429-1435, Nov 2009.

[18] A. Gubenko, D. Livshits, I. Krestnikov, S. Mikhrin, A. Kozhukhov, A. Kovsh, N. Ledentsov, A. Zhukov, and E. Portnoi, "High-power monolithic passively modelocked quantum-dot laser," Electron. Lett., vol. 41, pp. 1124-1125, 2005.

[19] M. A. Cataluna, E. A. Viktorov, P. Mandel, W. Sibbett, D. A. Livshits, J. Weimert, A. R. Kovsh, and E. U. Rafailov, "Temperature dependence of pulse duration in a mode-locked quantum-dot laser," Appl. Phys. Lett., vol. 90, pp. 101102-3, 2007.

[20] M. A. Cataluna, D. B. Malins, A. Gomez-Iglesias, W. Sibbett, A. Miller, and E. U. Rafailov, "Temperature dependence of electroabsorption dynamics in an InAs quantum-dot saturable absorber at $1.3 \mu \mathrm{m}$ and its impact on mode-locked quantum-dot lasers," Appl. Phys. Lett., vol. 97, pp. 121110-3, 2010

[21] A. Markus, J. X. Chen, C. Paranthoen, A. Fiore, C. Platz, and O. Gauthier-Lafaye, "Simultaneous two-state lasing in quantum-dot lasers," Appl. Phys. Lett., vol. 82, pp. 1818-1820, 2003.

[22] S. Schneider, P. Borri, W. Langbein, U. Woggon, R. L. Sellin, D. Ouyang, and D. Bimberg, "Excited-state gain dynamics in InGaAs quantum-dot amplifiers," IEEE Photon. Technol. Lett., vol. 17, pp. 2014-2016, 2005.

[23] T. Piwonski, J. Pulka, G. Madden, G. Huyet, J. Houlihan, E. A. Viktorov, T. Erneux, and P. Mandel, "Intradot dynamics of InAs quantum dot based electroabsorbers," Appl. Phys. Lett., vol. 94, pp. 123504-3, 2009.

[24] E. U. Rafailov, A. D. McRobbie, M. A. Cataluna, L. O'Faolain, W. Sibbett, and D. A. Livshits, "Investigation of transition dynamics in a quantum-dot laser optically pumped by femtosecond pulses," Appl. Phys. Lett., vol. 88, pp. 41101-3, 2006.
[25] M. A. Cataluna, E. U. Rafailov, A. D. McRobbie, W. Sibbett, D. A. Livshits, and A. R. Kovsh, "Ground and excited-state modelocking in a two-section quantum-dot laser," in $18^{\text {th }}$ Annual Meeting of the IEEE Lasers and Electro-Optics Society, Sydney, Australia, 2005, pp. 870-871.

[26] M. A. Cataluna, W. Sibbett, D. A. Livshits, J. Weimert, A. R. Kovsh, and E. U. Rafailov, "Stable mode locking via ground- or excited-state transitions in a two-section quantum-dot laser," Appl. Phys. Lett., vol. 89, pp. 81124-3, 2006.

[27] J. Kim, M.-T. Choi, and P. J. Delfyett, "Pulse generation and compression via ground and excited states from a grating coupled passively mode-locked quantum dot two-section diode laser," Appl. Phys. Lett., vol. 89, pp. 261106-3, 2006.

[28] S. Breuer, W. Elsasser, and M. Hopkinson, "State-switched modelocking of two-segment quantum dot laser via self-electrooptical quantum dot absorber," Electron. Lett., vol. 46, pp. 161$162,2010$.

[29] J. R. Liu, Z. G. Lu, S. Raymond, P. J. Poole, P. J. Barrios, and D. Poitras, "Dual-wavelength $92.5 \mathrm{GHz}$ self-mode-locked InP-based quantum dot laser," Opt. Lett., vol. 33, pp. 1702-1704, Aug 1 2008.

[30] M. A. Cataluna, D. I. Nikitchev, I. Krestnikov, D. A. Livshits, A. R. Kovsh, and E. U. Rafailov, "Dual-wavelength mode-locked GaAs-based quantum-dot laser," in CLEO-Europe, 2009, p. CB4_6.

[31] M. A. Cataluna, D. I. Nikitichev, S. Mikroulis, H. Simos, C. Simos, C. Mesaritakis, D. Syvridis, I. Krestnikov, D. Livshits, and E. U. Rafailov, "Dual-wavelength mode-locked quantum-dot laser, via ground and excited state transitions: experimental and theoretical investigation," Opt. Express, vol. 18, pp. 12832-12838, 2010.

[32] A. Leitenstorfer, C. Fürst, and A. Laubereau, "Widely tunable twocolor mode-locked Ti:sapphire laser with pulse jitter of less than 2 fs," Opt. Lett., vol. 20, pp. 916-918, 1995.

[33] S. Breuer, M. Rossetti, W. Elsasser, L. Drzewietzki, P. Bardella, I. Montrosset, M. Krakowski, and M. Hopkinson, "Reverseemission-state-transition mode locking of a two-section InAs/InGaAs quantum dot laser," Appl. Phys. Lett., vol. 97, pp. 071118-3, 2010.

[34] J. Kim and P. J. Delfyett, "Interband optical pulse injection locking of quantum dot mode-locked semiconductor laser," Opt. Express, vol. 16, pp. 11153-11161, Jul 212008.

[35] G. Ortner, C. N. Allen, C. Dion, P. Barrios, D. Poitras, D. Dalacu, G. Pakulski, J. Lapointe, P. J. Poole, W. Render, and S. Raymond, "External cavity InAs/InP quantum dot laser with a tuning range of 166 nm," Appl. Phys. Lett., vol. 88, pp. 121119-3, 2006.

[36] H. Kawaguchi, "Bistable laser diodes and their applications: state of the art," IEEE J. Sel. Top. Quant. Electron., vol. 3, pp. 1254$1270,1997$.

[37] M. Feng, N. A. Brilliant, S. T. Cundiff, R. P. Mirin, and K. L. Silverman, "Wavelength Bistability in Two-Section Mode-Locked Quantum-Dot Diode Lasers," IEEE Photon. Tech. Lett., vol. 19, pp. 804-806, 2007.

[38] F. Mingming, S. T. Cundiff, R. P. Mirin, and K. L. Silverman, "Wavelength Bistability and Switching in Two-Section QuantumDot Diode Lasers," IEEE J. Quant. Electron., vol. 46, pp. 951958, 2010.

[39] M. Kuramoto, N. Kitajima, H. Guo, Y. Furushima, M. Ikeda, and H. Yokoyama, "Two-photon fluorescence bioimaging with an allsemiconductor laser picosecond pulse source," Opt. Lett., vol. 32, pp. 2726-2728, 2007.

[40] M. T. Choi, W. Lee, J. M. Kim, and P. J. Delfyett, "Ultrashort, high-power pulse generation from a master oscillator power amplifier based on external cavity mode locking of a quantum-dot two-section diode laser," Appl. Phys. Lett., vol. 87, pp. 221107-3, Nov 2005.

[41] A. D. McRobbie, M. A. Cataluna, S. A. Zolotovskaya, D. A. Livshits, W. Sibbett, and E. U. Rafailov, "High power allquantum-dot-based external cavity modelocked laser," Electron. Lett., vol. 43, pp. 812-813, 2007.

[42] M. Xia, M. G. Thompson, R. V. Penty, and I. H. White, "ExternalCavity Mode-Locked Quantum-Dot Lasers for Low Repetition 
Rate, Sub-Picosecond Pulse Generation," 2008 Conference on Lasers and Electro-Optics \& Quantum Electronics and Laser Science Conference, Vols 1-9, pp. 1137-1138, 2008.

[43] Y. Ding, D. I. Nikitichev, I. Krestnikov, D. Livshits, M. A. Cataluna, and E. U. Rafailov, "Quantum-dot external-cavity passively modelocked laser with high peak power and pulse energy," Electron. Lett., vol. 46, pp. 1516-1518, 2010.

[44] M. G. Thompson, A. Rae, R. L. Sellin, C. Marinelli, R. V. Penty, I. H. White, A. R. Kovsh, S. S. Mikhrin, D. A. Livshits, and I. L. Krestnikov, "Subpicosecond high-power mode locking using flared waveguide monolithic quantum-dot lasers," Appl. Phys. Lett., vol. 88, pp. 133119-3, 2006.

[45] E. A. Viktorov, P. Mandel, M. Kuntz, G. Fiol, D. Bimberg, A. G. Vladimirov, and M. Wolfrum, "Stability of the mode-locked regime in quantum dot lasers," Appl. Phys. Lett., vol. 91, pp. 231116-3, Dec 32007

[46] T. W. Berg and J. Mork, "Quantum dot amplifiers with high output power and low noise," Appl. Phys. Lett., vol. 82, pp. 30833085, 2003.

[47] P. Vasil'ev, Ultrafast Diode Lasers: Fundamentals and Applications. Boston: Artech House, 1995.

[48] G. Carpintero, M. G. Thompson, R. V. Penty, and I. H. White, "Low Noise Performance of Passively Mode-Locked 10-GHz Quantum-Dot Laser Diode," IEEE Photon. Technol. Lett., vol. 21, pp. 389-391, Mar 152009.

[49] M. Laemmlin, G. Fiol, C. Meuer, M. Kuntz, F. Hopfer, A. R. Kovsh, N. N. Ledentsov, and D. Bimberg, "Distortion-free optical amplification of $20-80 \mathrm{GHz}$ modelocked laser pulses at $1.3 \mu \mathrm{m}$ using quantum dots," Electron. Lett., vol. 42, pp. 697-699, 2006.

[50] A. Mar, R. Helkey, W. X. Zou, D. B. Young, and J. E. Bowers, "High-power mode-locked semiconductor lasers using flared waveguides," Appl. Phys. Lett., vol. 66, pp. 3558-3560, 1995.

[51] D. Nikitichev, Y. Ding, M. Ruiz, M. Calligaro, N. Michel, M. Krakowski, I. Krestnikov, D. Livshits, M. Cataluna, and E. Rafailov, "High-power passively mode-locked tapered InAs/GaAs quantum-dot lasers," Appl. Phys. B, pp. 1-5, 2010. 


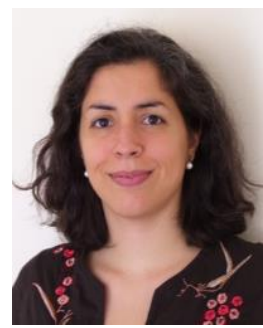

Maria Ana Cataluna graduated in Physics from the Instituto Superior Técnico, Lisbon, Portugal (2001), where she was a Research Assistant from 2000 to 2002. In 2003, she moved to the University of St. Andrews, UK, and was awarded the Ph. D. in Laser Physics in 2007. Since 2007 she has been with the University of Dundee, UK - firstly as a Research Fellow, and since 2009 as a Lecturer and Royal Academy of Engineering/EPSRC Research Fellow. Dr Cataluna is a Work Package Leader in the European project FAST-DOT, which aims to develop compact and low-cost ultrafast quantum-dot lasers for biophotonics. She has authored/co-authored more than 50 papers in peer-reviewed journals and conferences, three invited book chapters and one book. She was the recipient of an International $\mathrm{PhD}$ Studentship from Fundação para a Ciência e Technologia (Portugal) in 2003, the IEEE Photonics Society Graduate Student Fellowship in 2007, and a Royal Academy of Engineering/EPSRC Research Fellowship in 2009. Her current research interests include the physics and technology of ultrafast and broadly tunable semiconductor lasers and related applications.

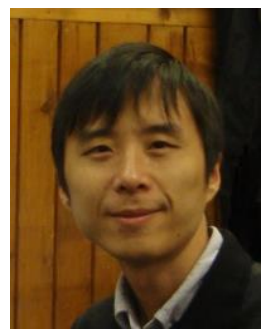

Ying Ding received the Ph.D. degree in microelectronics and solid state electronics from the Institute of Semiconductors, Chinese Academy of Sciences, Beijing, China.

From 2005 to 2008, he was in the RCIQE, Hokkaido Univ., Japan as a Postdoctoral Researcher, where he was engaged in research on semiconductor nanowires. In 2008, he joined the Nanyang Technological Univ., Singapore as a Research Fellow, where he worked on quantum-dot verticalcavity surface-emitting lasers (QD-VCSELs). He is currently a Research Fellow at the Univ. of Dundee, UK. His current research interests include external-cavity mode-locked quantum-dot lasers and high-power integrated light sources with ultra-short pulse generation.

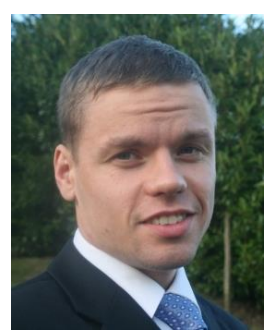

Daniil Nikitichev received Master degree in State University of the Information Technologies, Mechanics and Optics, Saint Petersburg, Russia, in 2008. He was trained at the State Optic Institute during the year 2006. He worked as an engineer at the Central Research Institute of Structural Materials "Prometey", doing research work in the area of Laser Thermo strengthening from 20062008. He is currently a PhD student at the University of Dundee, where he is working on the FAST-DOT project, where he is engaged with the development of a new generation of compact and low cost ultrafast lasers based on quantum-dot materials.

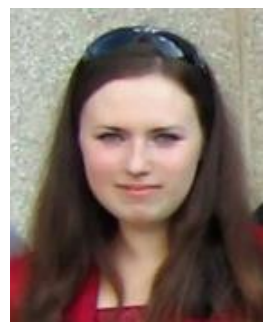

Ksenia Fedorova received the B.Sc. and M.Sc. degrees in physics from the Nizhny Novgorod State University named after N.I. Lobachevsky, Nizhny Novgorod, Russia, in 2005 and 2007, respectively. She is currently working toward the Ph.D. degree at the Department of Electronic Engineering and Physics, University of Dundee, Dundee, U.K.

Her research project is centred on the study of novel quantum-dot materials for compact broadly tunable lasers and non-linear optics. Currently, her research interests include the generation of mid-IR radiation using novel quasi-phasematched semiconductor nonlinear crystals based on GaAs and wavelength conversion using periodically poled nonlinear crystals and quantum-dot edgeemitting laser diodes.

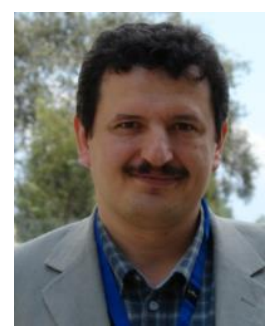

Edik Rafailov received the Ph.D. degrees from the Ioffe Institute, St Petersburg in 13.01.1992. In 1997 he moved from St Petersburg to St Andrews University as a Research Fellow and in 2005 he moved to Dundee University as a lecturer and established new Photonics and Nanoscience group. In 2008 he became a Reader and in 2010 Professor. He has authored and co-authored over 200 articles in refereed journals and conference proceedings, including one book, three invited chapters and numerous invited talks to CLEO, SPIE and LEOS. He also holds 8 UK and two US patents. Prof. Rafailov coordinates a $€ 13.7 \mathrm{M}$ FP7 European IP project (FAST-DOT) intended to develop new miniature low-cost ultrafast lasers based on quantum-dot materials for applications in biophotonics and cellular-surgery. He also leads a few others projects funded by FP7 EU and EPSRC. His current research interests include novel high-power $\mathrm{CW}$, short, ultrashort-pulse and high-repetition rate lasers; generation of $\mathrm{UV} /$ visible/IR/MIR and $\mathrm{THz}$ radiation, nano-structures; nonlinear and integrated optics; biophotonics. 